\title{
Interaction Design Based on Relieving Psychological Pressure of "996" Group
}

\author{
Xiaoao Dong ${ }^{1, *}$ \\ ${ }^{1}$ School of design and innovation of CAA, China Academy of Art, Hangzhou, Zhejiang, 310052, China
}

\begin{abstract}
In recent years, the phenomenon of "996" has attracted extensive concern from the society. The rapid enterprise development requires more time and work from employees, which cause conflict with their family life and lays a hidden danger to their physical and mental health. A lot of useful exploration on how to relieve the pressure of "996" have been conducted, but no convenient and effective methods have been found. In respect of the characteristics of the "996" group, the author utilizes the advantages of interactive art, integrates the sympathetic design concept of intelligent interactive equipment with the breathing decompression method by studying and applying Processing and Arduino, which enables people to actively apply the decompression device in a relaxed and natural state, release pressure anytime and anywhere. This is a new exploration of the integration of interactive art and daily life, aiming to provide new ideas for the integration of interactive art into the daily life of the public and problem solving.
\end{abstract}

\section{The characteristics and pressure of "996" group}

On December 2, 2019, the National Language Resources Monitoring and Research Center released the "Top 10 Internet Words of 2019", with "996" among them. On the same day, "996" was selected as one of the "Top 10 Buzzwords of 2019" by Yaowenjiaozi magazine[1]. "996" has directly led to an irregular life, including working overtime, staying up late, eating irregularly, lack of exercise, in a state of sub-health and even cardiovascular disease and other serious problems. Long time highpressure life causes mental health problems and negative emotions, which affect people's work and family relations, even cause a vicious circle. The quality of life is seriously reduced.

The "996" phenomenon emerged with the development of the Internet economy. Most of the "996" group are IT technicians and senior white-collar workers. Their jobs are well paid, but their work is also stressful and high technical demanding. Overtime working is regular and generally required. In recent years, the frequent occurrence of extreme phenomena such as sudden death of employees in Internet companies has aroused great concern of the society.

The "996" mode is constantly criticized and many experts as well as scholars criticize the "996" mode even from the legal level. However, in the real society, it is often difficult for people to really get rid of "996" mode under the pressure of life and the pursuit of ideals. The "996" working mode cannot be completely abandoned in a short time. Therefore, how to effectively alleviate the physical and mental pressure of this group is worth studying.

\section{Exploring the ways to reduce stress}

\subsection{Common ways to reduce stress}

Stress is a common problem in modern fast-paced life. People also try to decompress in a variety of ways. The common ways of decompression include followings:

- Sleeping. People can relieve their mental tension and get relaxed after a long sleep.

- Taking social activities. People can reduce their pressure by communicating with other people, seek support and learn from other people's experience to broaden their thinking.

- Shopping. The stimulation of new things and satisfaction brought by shopping their favorite stuffs will make people forget the trouble and reduce the stress.

- Eating. People in anxiety or depression can release emotions by eating favorite food. The stimulation of teeth chewing can also relieve psychological pressure in a certain extent.

- Sporting. Doing sports is a popular way for people to reduce their stress. Any sports, say swimming, running, playing balls or extreme sport can help people to release their pressure from physically or mentally.

\subsection{Exploration of pressure release approaches for "996" group}

With the continuous overtime work, it is difficult for the "996" group to achieve the pressure release through above common ways because they don't have enough time for 
sleeping, socializing or shopping. It is difficult for them to have a complete time for relaxation. Most people seldom disclose their pressure to their relatives and friends, and only a few people can develop the habit of exercising. It is very important to find a more easy, convenient and effective way to divert attention and relieve the psychological stress by utilizing their short free time.

Based on the characteristics of the "996" group, it is more practical to explore a natural and relax emotional device, which can be applied in the working environment and lead the active participation in "996" group's daily life by combining the concept of interactive design and emotional design.

Most of the Internet companies are located in the big cities and lack the breath of nature, thus we can create a natural atmosphere by utilizing the forest. Different colors have different emotional semantics, say green can impress a person's perception of peace and comfort and the wavelength of green light in the center of the visible light wavelength range, which is the frequency of colors that the human eye is more sensitive and easy to receive, and not easy to fatigue. We can set green as the main tonal of the pressure release devices, which contribute to a better relax for the "996" group of long-term working on the computer screen.

\subsection{Breathing to help reduce stress}

Deep breathing is one of the most effective ways to relieve stress and restore calm. It can be done anywhere without any conditions. The state of mind and how brain deals with stress are all affected by breathing. People who feel nervous, stressed, or helpless often take deep breaths for relief and relaxation. When they take a deep breath, they shift their attention from the mind to their bodily sensations, thereby diverting their attention from their anxiety.

But the benefits of deep breathing go beyond distraction. When we face problem or feel frustrated, we all feel calmer when slowing down our breathing. Some scientists even call deep breathing the "mental and physical reset." Gordon, professor at School of Medicine in Georgetown University, has written that "deep, slow breathing is probably the most effective treatment for stress[2]." Breathing in a lot of fresh air makes people feel refreshed, slows heart rate, lowers blood pressure, and puts people in a new state to work.

\subsection{Music therapy to help reduce stress}

"Sentiment cure" of traditional Chinese medicine believes that people who are emotionally excited should listen to peaceful and sad music and people who are emotionally depressed should accept cheerful music to change their original state of mind[3]. Music can influence the emotions, then affect the body. Music with different melodies can make people feel excited or relaxed, reduce heart rate and blood pressure to a certain extent, and enhance people's thinking, reasoning and memory ability. Light music can relax the mood to appease the mind and make the impatient mood calm and stable. Serious classical music, with its elegant melodies and thoughtprovoking sounds, can clear the mind and get rid of anxiety. For example, "Gentle Day" is graceful and soothing, close to nature, which makes people relax and feel happy. "The Right Path" has a brisk rhythm, bringing anxious and depressed people out of the dull mood. Folk music "flying a kite" is classical and elegant, making people feel easy and comfort.

In this study, breathing method and music therapy will be applied in interactive devices. The device will make "996" group participating deeply and get their stress naturally released by taking the breathing method as the main approach, together with music and environment.

\section{Interaction design}

\subsection{Brief introduction to interaction design}

Interaction design itself is a humanistic undertaking. It's People interacting with products and services. The primary goal of interaction design is to meet people's needs and desires[4]. With the development of modern science and technology, there are more and more emerging products and interactive ways, and people have realized the importance of interactive experience. Interaction design should start from the user's point of view and consider how to make the product simple, convenient and effective. It should have a deep understanding of the user's expectations, observe the behavior of the user when interacting with the product, gain insight into the psychological characteristics of "people", and enhance and expand them.

\subsection{Application of Processing programming technology in interactive devices}

With the appearance of Processing, programming is no longer the patent of program technicians. Instead, artists can make use of Processing for artistic creation, which broadens the boundary of art and greatly improves the efficiency of artistic creation. Data of sound and light can be transformed into interesting artistic images. Processing can also interact with the physical world through communication with external devices. Using Processing in interactive devices can increase the audience's participation and immersion, increase their interests, encourage users to get interested in interactive devices and further involvement, feel the true self in the interaction, and have a dialogue with themselves.

Arduino is today's most popular collection of open source hardware and software development platform. A rich variety of sensors enable Arduino to experience and interact with the physical world in a variety of interesting ways. Processing and Arduino both share a common programming environment and similar syntax. The combination of the two technologies better integrates electronic technology, single-chip technology and graphic programming technology, making programming design more humanized. The digital signal or analogue information sent by the sensor is converted into data by the Arduino board. Processing reads these data through the 
serial port and presents them on the computer screen in real time after processing them through relevant functions[5]. The data can be expressed in a variety of ways, including patterns, sound or video, to enable the users to interact with the device.

\section{The stress relief experiment by interactive device, taking "Breathing" as an example}

\subsection{Designing concept}
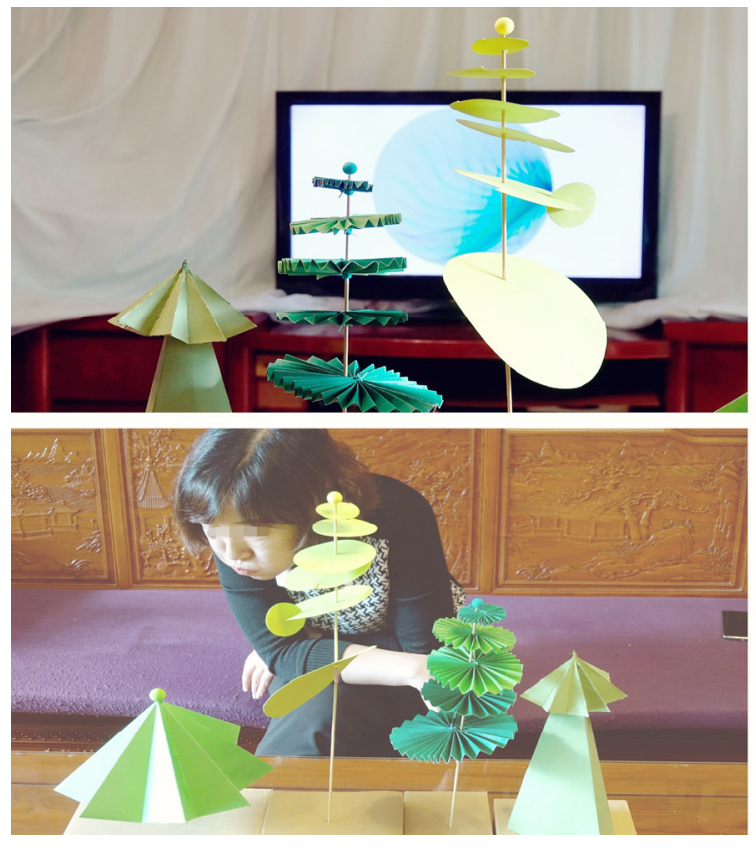

Figure 1. Design and experience the device

\subsection{Technical analysis and practice}

This interactive device uses Arduino chip and various components to control the whole operation and applies Processing to draw artistic patterns and interact with experiencers.

It uses pressure sensors and MP3 modules and is programmed to interact with the user. The four canopies represent different emotional meanings: relaxed \&
Experiment device "Breath" attempts to solve the pressure problem of "996" group in a more natural and convenient way, by using the origami crown, breathing stress relief method, music therapy and art programming, interactive technology. The device tries to guide the "996" group to actively release pressure, feeling the pressure release in the deep breathing, calming the mood in the music interaction, feeling and making dialogue with themselves. The device can be placed in the office area or rest area, etc., without restriction of place. The "996" group can use the device at any time during work, and feel the pressure relief anytime and anywhere.
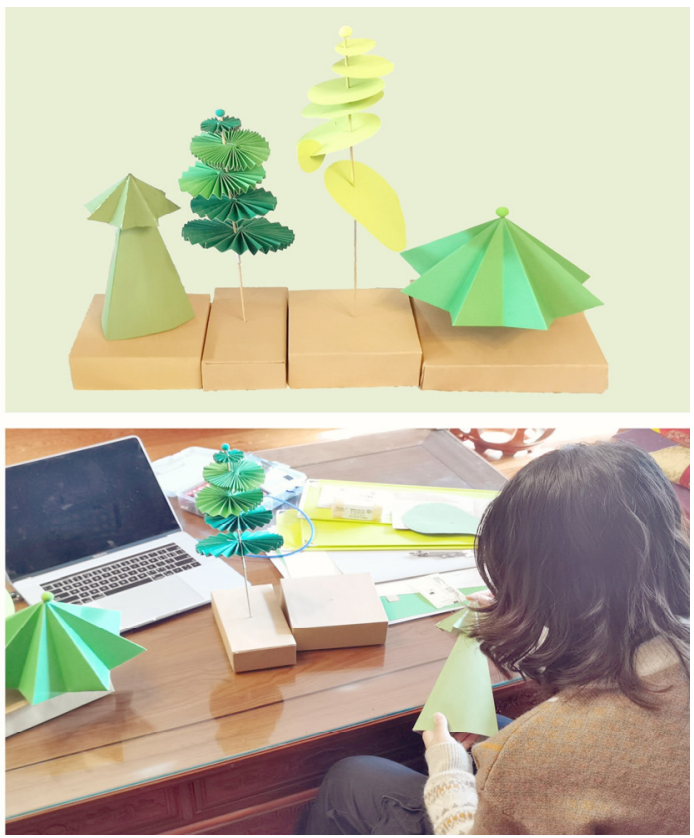

gentle, soothing, cheerful and quiet \& serious, which correspond to the types of pressure that the "996" group generally faces: tension, anger \& irritability, disorder, sadness, depression and loneliness. Experiencers blow on the crown of any tree, and when the crown rotates, music with corresponding emotional meaning will be released to alleviate different types of psychological pressure of the "996" group. The MP3 module controls the sound, and the pressure sensor senses the blowing pressure. When the pressure exceeds the specified size, the trigger device releases the corresponding music. 


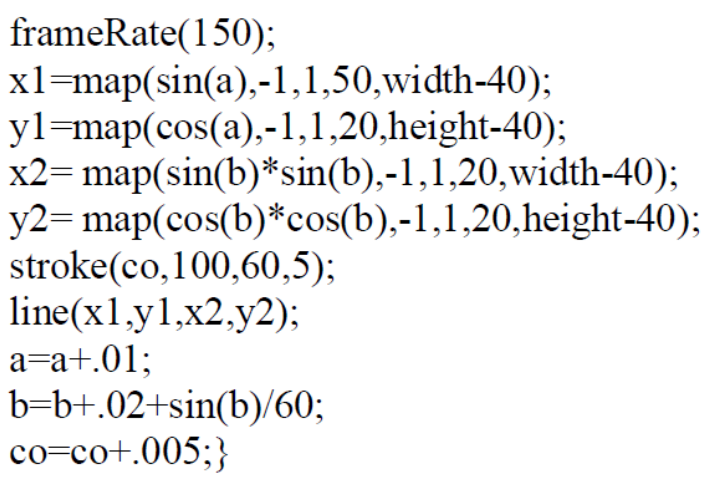

Figure 4. Code setting for controlling pattern drawing rate by sensing blowing pressure

\section{Summary}

With the development of the times, the expression of design is gradually combined with information technology and emphasizes more on the interaction between people and design. Interaction design is playing an increasingly important role in modern society. People attach more and more importance to digital technology, and digital media is entering People's Daily life and production at an unprecedented speed, affecting people's life style. Interaction device "Breath" actively explores the role of interaction design device in alleviating psychological pressure for "996" group in the new era, and provides new ideas for the integration of interaction design and the times. During the experiment, most experiencers comment that the device has a good effect on alleviating pressure. Interaction design should not only stay at the artistic level, but also be integrated into daily life. We should explore and study how to use interaction design to solve practical problems in a more comprehensive and creative way.

\section{References}

1. Yang, Y.M. Escape from "996", individuals, enterprises and the law should do something[N]. China Business Daily, 2021-1-11(A07).

2. Wang, Z.C. (2010) Learn to Deep Breathe. Health Club, 9: 44.

3. Zhang, Z. (2010) The music of anger mitigation. Journal of Changchun University, 11: 122-123.

4. Cooper, A., Reimann, R., Cronin, D., Noessel, C., Csizmadi, J., LeMoine, D. (2015) About Face 4: The Essentials of Interaction Design. Electronic Industry Press, Beijing.

5. Chen, X.W., Li, J.L. (2020) The Art of Programming in Digital Media Interaction. Art Education, 3: 130133. 